\title{
Sharpening Interpolated Image by Using Cube of Pixel Difference
}

\author{
Naoki ONO*, Kiichi URAHAMA \\ Faculty of Design, Kyushu University, \\ Shiobaru 4-9-1, Minami-ku, Fukuoka 815-8540 Japan \\ *Corresponding Author: ono@design.kyushu-u.ac.jp
}

\begin{abstract}
In order to improve image resolution, any interpolation has to be applied. However, in general, interpolation generates smooth connections between adjacent sampling points and yields blurred edges in the interpolated image. To suppress such a blur in edges, it is desired to include any sharpening effect in the interpolating process. In this paper, we propose a sharpening method using cubic operations of the differences of pixel values. Experimental results show that this method produces sharp high resolution image which is more similar to a target image than that by any other method.
\end{abstract}

Keywords: interpolation, high resolution, unsharp, cubic operation.

\section{Introduction}

Improving image resolution is a process for making a high resolution image from a low resolution image. In order to improve image resolution, any interpolation has to be applied. However, in general, interpolation generates smooth connections between adjacent sampling points and yields blurred edges in the interpolated image. To suppress such a blur in edges, it is desired to include any sharpening effect in the interpolating process.

For sharping images, linear filters such as Laplacian operator, unsharp masking based on Gaussian filter are conventional methods and are often used in general. A cubic unsharp masking method is proposed by G. Ramponi [1][2]. The method uses an operator which consists of the product with Laplacian and a quadratic function of the local gradient. The cubic unsharp masking method performs effectively for the images with noise since the method suppresses the noise enhancement. However, for a noiseless image the method may not produce better result than any other sharpening method.

For producing a high resolution image with high frequency components, a method using cube of high pass filter is proposed [3]. In the paper, the cube of Laplacian filter is applied to video images to produce high resolution images of them and the validity of the method for producing high frequency components are shown.

In this paper, we propose a sharpening method which is not cube of linear filter but uses weighted sum of cubic operations of the differences between a value of pixel and those of the surrounding pixels. Experimental results for an interpolated image show that this method produces sharp high resolution image which is more similar to a target image than that by any other methods.

\section{Sharpening for interpolated image}

\subsection{Unsharp masking by liner operation}

The fundamental idea of unsharp mask (UM) is to add high frequency components to the input image. The high frequency components of an input image is obtained by subtraction of a low pass filtered version of the input image from the input image itself.

Given interpolated image $d_{i j}(i=1, \cdots, M ; j=1, \cdots, N)$, an unsharp masked image $f_{i j}$, which is derived by the standard unsharp masking with linear low pass filter, is given by

$$
f_{i j}=d_{i j}+\delta\left(d_{i j}-\tilde{d}_{i j}\right),
$$

where $\tilde{d}_{i j}$ is a low pass filtered version of $d_{i j}$.

Using Gaussian filter

$$
w_{l m}=\frac{e^{-\alpha\left(l^{2}+m^{2}\right)}}{\sum_{l=-p}^{p} \sum_{m=-p}^{p} e^{-\alpha\left(l^{2}+m^{2}\right)}},
$$


for making a low pass filtered image $\tilde{d}_{i j}$, the UM produces an enhanced image $f_{g i j}$ expressed by

$$
\begin{aligned}
f_{g i j} & =d_{i j}+\delta\left(d_{i j}-\tilde{d}_{i j}\right) \\
& =d_{i j}+\delta\left(d_{i j}-\sum_{l=-p}^{p} \sum_{m=-p}^{p} w_{l m} d_{i+l, j+m}\right), \\
& =d_{i j}+\delta \sum_{l=-p}^{p} \sum_{m=-p}^{p} w_{l m}\left(d_{i j}-d_{i+l, j+m}\right)
\end{aligned}
$$

where $\alpha(>0)$ and $p$ are parameters which control smoothness and size of mask respectively. Furthermore we control the sharpness by a parameter $\delta(>0)$.

\subsection{Sharpening by cube of liner masking}

If we adopt the cube of subtraction from the input image a low pass filtered version as the high frequency components, slight changes in the image can be enhanced and detail information becomes more visible effectively. According to this idea, enhanced image $f_{c i j}$ is produced by

$$
\begin{aligned}
f_{c i j} & =d_{i j}+\delta\left(d_{i j}-\tilde{d}_{i j}\right)^{3} \\
& =d_{i j}+\delta\left(d_{i j}-\sum_{l=-p}^{p} \sum_{m=-p}^{p} w_{l m} d_{i+l, j+m}\right)^{3} . \\
& =d_{i j}+\delta\left(\sum_{l=-p}^{p} \sum_{m=-p}^{p} w_{l m}\left(d_{i j}-d_{i+l, j+m}\right)\right)^{3}
\end{aligned}
$$

This method is, so to speak, the cube of UM. Using the cube of Laplacian as the high frequency component instead of second term in eq.(4), the sharpening procedure is equivalent to that in [3].

\subsection{Sharpening by using cube of pixel differences}

We propose a sharpening method which is not cube of linear filter but uses weighted sum of cubic operations of the differences between a value of pixel and those of the surrounding pixels, i.e.

$$
f_{d i j}=d_{i j}+\delta \sum_{l=-p}^{p} \sum_{m=-p}^{p} w_{l m}\left(d_{i j}-d_{i+l, j+m}\right)^{3} .
$$

This method also enhanced slight changes effectively. However, since the value of improvement often becomes extremely large, we suppress the value of improvement by setting a limit value $L$. Needless to say, the limitation should be applied to the cube of UM with eq.(4).

\section{Experiments}

\subsection{Sharpening image}

An original image consists of $256 \times 256$ pixels with 256 gray levels. Firstly, we interpolated the input image into an enlarged image $(512 \times 512$ size $)$ by the bilinear interpolation. Figure 1 shows the interpolated image. Appling the three sharpening methods to the interpolated image, we had three enhanced images. In the experiment, we set the parameters $p=2, \alpha=0.5, \delta=0.4$ and $L=4$.

We compared the proposed method with a conventional linear UM and the cube of UM experimentally. Figure 2, Fig. 3 and Fig. 4 show improved images by the linear UM, the cube of UM and the proposed method respectively. The image by the proposed method shows clear details of textures with suppressing excessive edge enhancement. Although the linear UM also gave clear texture details, all edges are enhanced too much. The cube of UM lost the texture details especially on a hat.

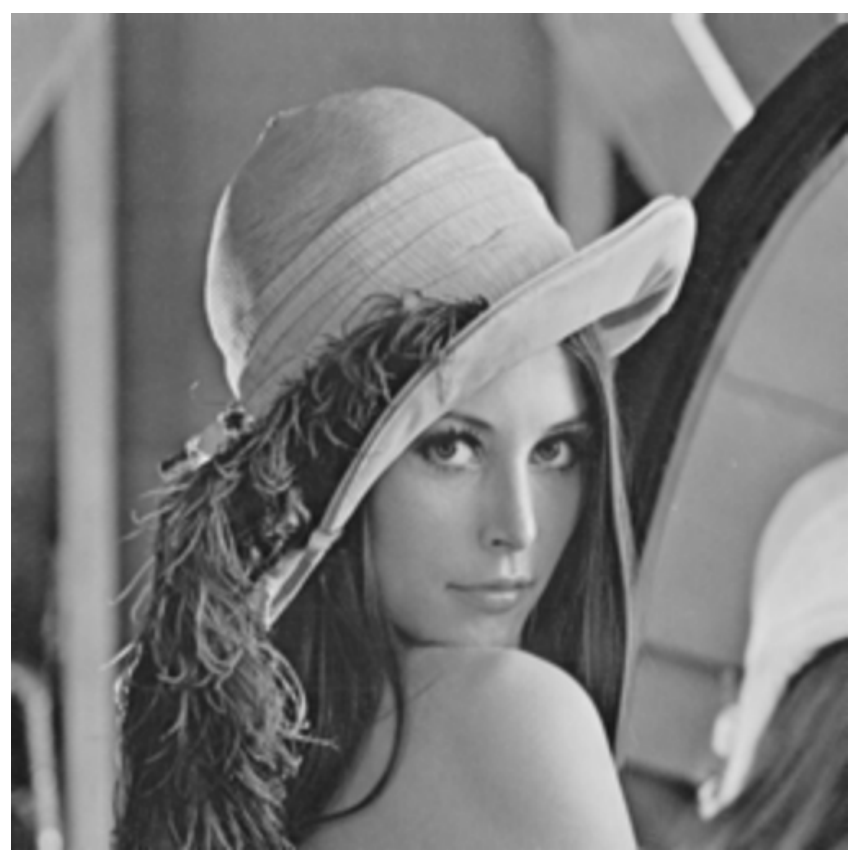

Fig.1. Bilinear interpolated image.

\subsection{Comparison on the similarities of local complexity}

In general, a performance of image processing is evaluated by using PSNR. However PSNR does not always show a reasonable value especially on sharpness of images. Since we now focus on the sharpness and enhancement of image details, we use the local complexity to evaluate the performance.

We employ the local standard deviation as the local complexity. Assuming that the local standard deviation of an enhanced image and that of the target image are represented by $s_{i j}$ and $s_{0 i j}$ respectively, the sum of difference of local complexities between the enhanced image and the target image is obtained by 


$$
S_{d}=\frac{1}{M N} \sum_{i=1}^{M} \sum_{j=1}^{N}\left(s_{i j}-s_{0 i j}\right)^{2} .
$$

The value derived by (6) gives the similarity between an enhanced image and a target image in the meaning of local complexity. The smaller $S_{d}$ is, the more similar the enhanced image and the target image are. In this experiment, we used a high resolution $512 \times 512$ image shown in Fig. 5 as the target image, where this image is the true high resolution image of the input $256 \times 256$ image. Standard deviations $s_{0 i j}$ for the target image and $s_{i j}$ for created images shown in Fig. 2, 3, 4 are calculated with $7 \times 7$ square window.

Table 1 shows the sum of difference of local complexities between the enhanced image and the target image by (6). $S_{d}$ for resultant images by the liner UM and the cube of UM are larger than $S_{d}$ for the bilinear interpolated image which is before enhancement. Especially the difference of the linear UM is much larger. These mean that the liner UM and the cube of UM degraded the similarity of local complexity for the bilinear interpolated image. $S_{d}$ for the proposed method is smallest in this experiment. This shows that the proposed method improved the interpolated image and gave most similar image to the target image at the point of complexity.
Table 1. The similarities of local complexity

\begin{tabular}{|l|c|}
\hline & $S_{d}$ \\
\hline Bilinear interpolation & 12.148497 \\
\hline Linear UM $\alpha=0.5, \delta=0.4$ & 42.288730 \\
\hline Cube of UM $\alpha=0.5, \delta=0.4, \mathrm{~L}=4$ & 27.291751 \\
\hline Proposed method $\alpha=0.5, \delta=0.4, \mathrm{~L}=4$ & 6.127024 \\
\hline
\end{tabular}

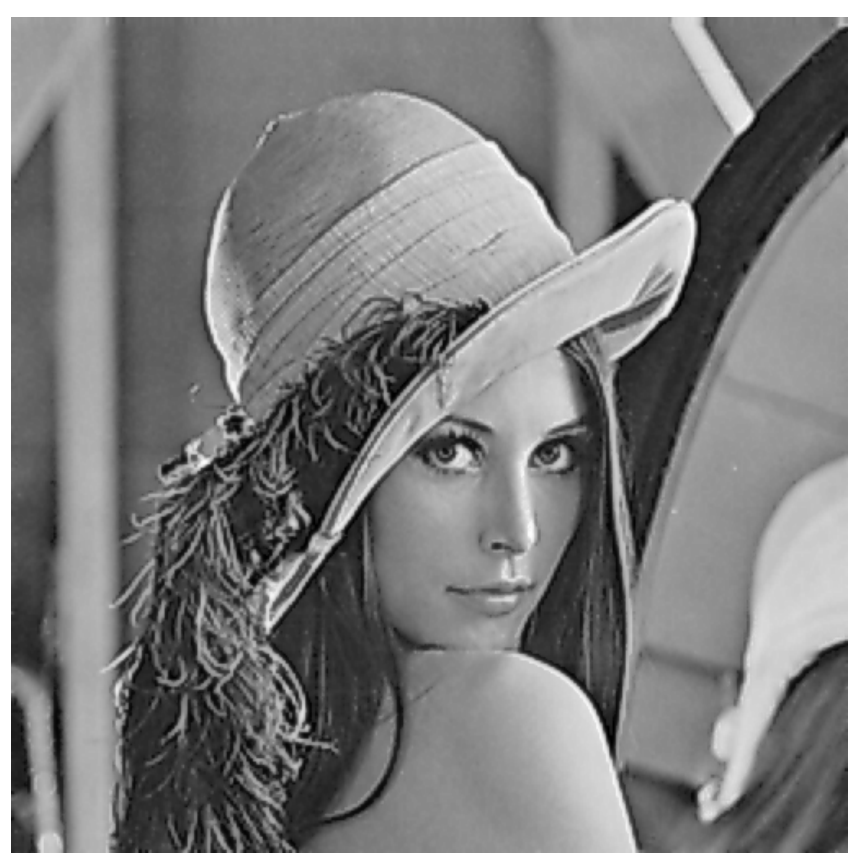

Fig.3. Result by Cube of UM.

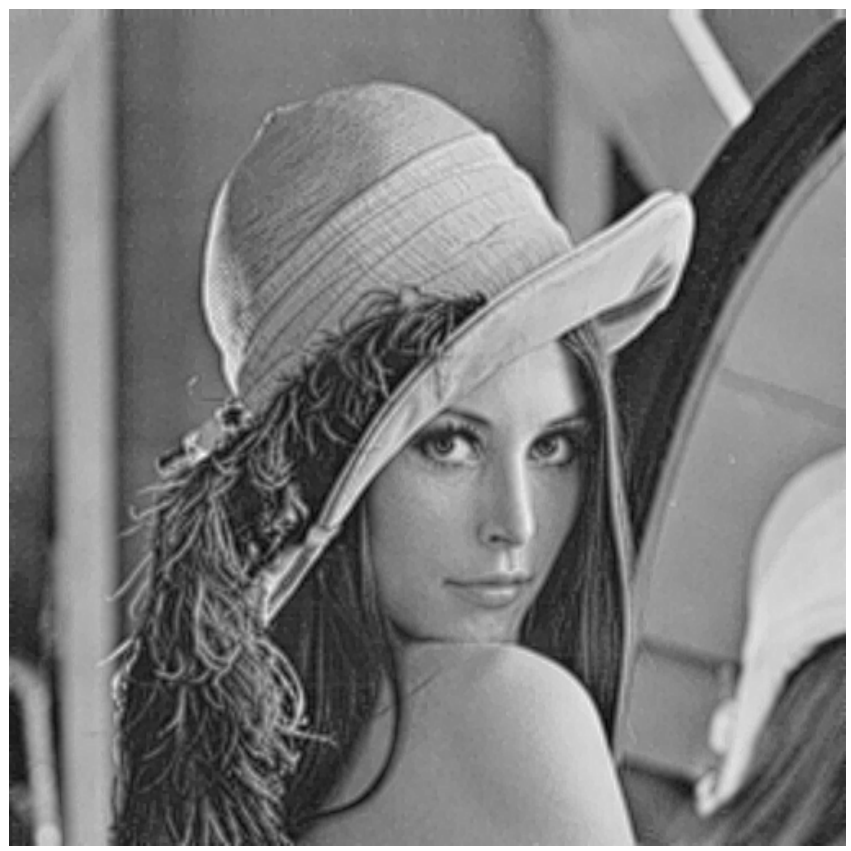

Fig.4. Result by the proposed method. 


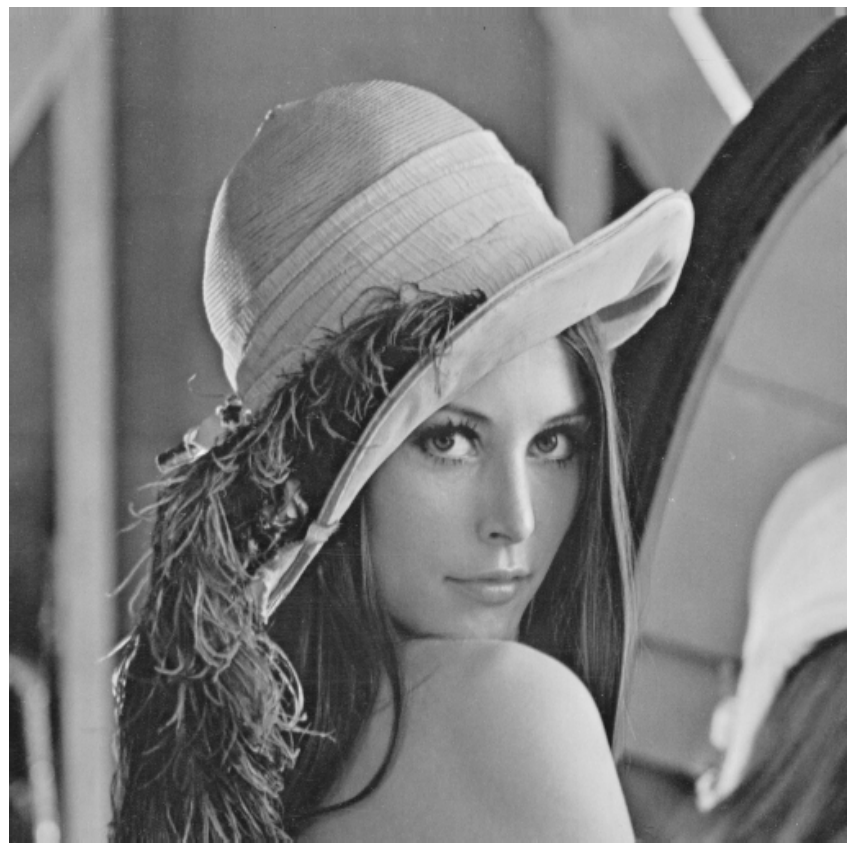

Fig.5. A target image.

\subsection{Comparison in the frequency domain}

By improving the resolution of image, the components in higher frequency domain are newly created. We evaluate the components in the low frequency domain included in the original low resolution image and those in the high frequency domain generated by the interpolation respectively.

After improving the image, it is desired to preserve the frequency components included in an input image before interpolation in order not to change the original image information. On the hand, the high frequency components which is not contained before interpolation should be create effectively to give a better impression as a high precision image.

Figure 6 shows the FFT result of the original low resolution image. Although the frequency domain of the original image is $256 \times 256$, the components are superimposed in the domain of $512 \times 512$ to compare with the interpolated high resolution images. Figure 6 also shows the original image does not have high frequency components in the outside of $256 \times 256$ low frequency domain.

Figure 7 shows the difference of frequency components between the bilinear interpolated image shown in Fig. 1 and the input low resolution image. Needless to say, difference in the outside of $256 \times 256$ is newly created high frequency components. Figure $8,9,10$ show that the difference between frequency components of Fig. 2, 3, 4 and that of Fig. 6 respectively. We evaluated whether the method creates high frequency components with suppressing the change in $256 \times 256$ low frequency components.

Although the bilinear interpolated image has new high frequency components in Fig.7, these components are yielded by the linear interpolation process and are not created from the characteristic of the input image.

From Fig. 8 and 9, although enhanced images by the linear UM and the cube of UM have high frequency components, components in low frequency domain have changed remarkably. This shows that the information of original image was changed obviously. Furthermore, the differences in low frequency domain for resultant images by the liner UM and the cube of UM are larger than those for the bilinear interpolated image which is before enhancement. This mean that the liner UM and the cube of UM distorted the original information from the bilinear interpolated image.

Figure 10 shows that the result by the proposed method have high frequency components and the difference of components in $256 \times 256$ low frequency domain are smaller than those of other resultant images. From this experiment, the proposed method yields high frequency components with suppressing change of information of original image reasonably.

\section{Conclusions}

In this paper, we proposed a sharpening method which is not cube of linear filter but uses weighted sum of cubic operations of the differences between a value of pixel and those of the surrounding pixels.

We applied some enhancement procedures for a linear interpolated image and compared the similarities of local complexity of the resultant images and a target image experimentally. The proposed method improved the interpolated image and gave most similar image to the target image at a point of complexity. We also evaluated the resultant images in the frequency domain. The proposed method yielded high frequency components with suppressing change of information of original image reasonably.

Experimental results for an interpolated image show that the proposed method produces sharp high resolution image which is more similar to a target image than that by any other method. 


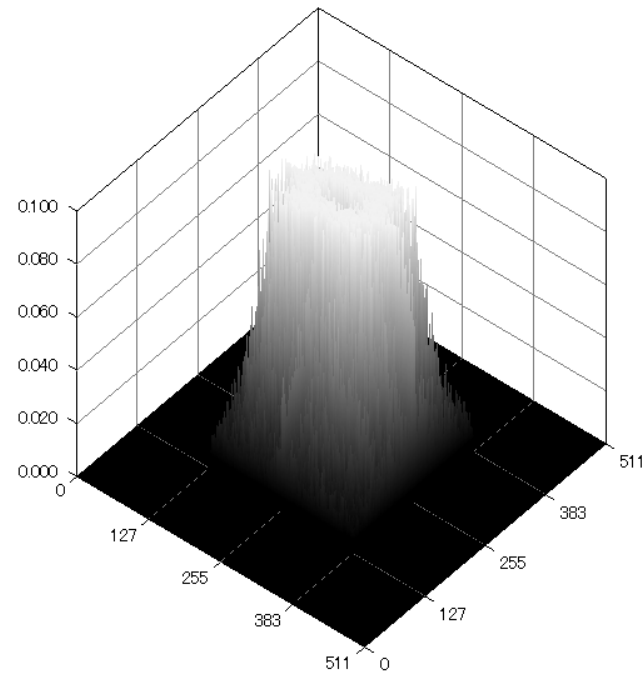

Fig.6. Power spectrum of the original image.

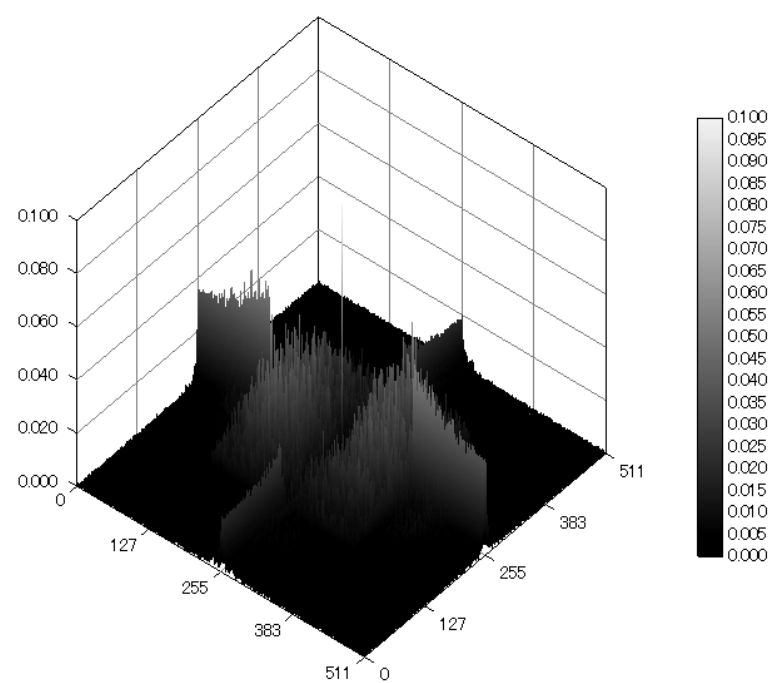

Fig.7. Difference of power spectrum between a bilinear interpolated image and the original image.

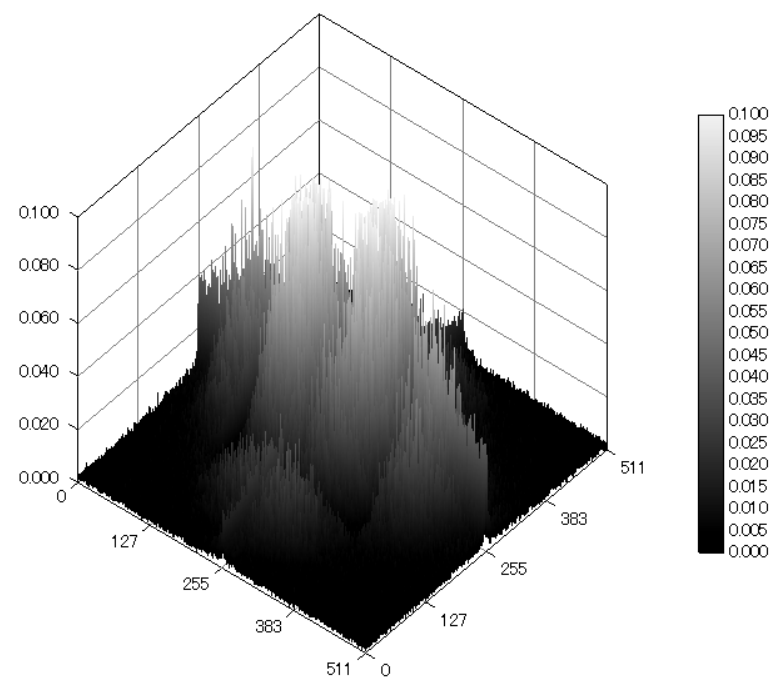

Fig.8. Difference of power spectrum between a linear UM image and the original image.

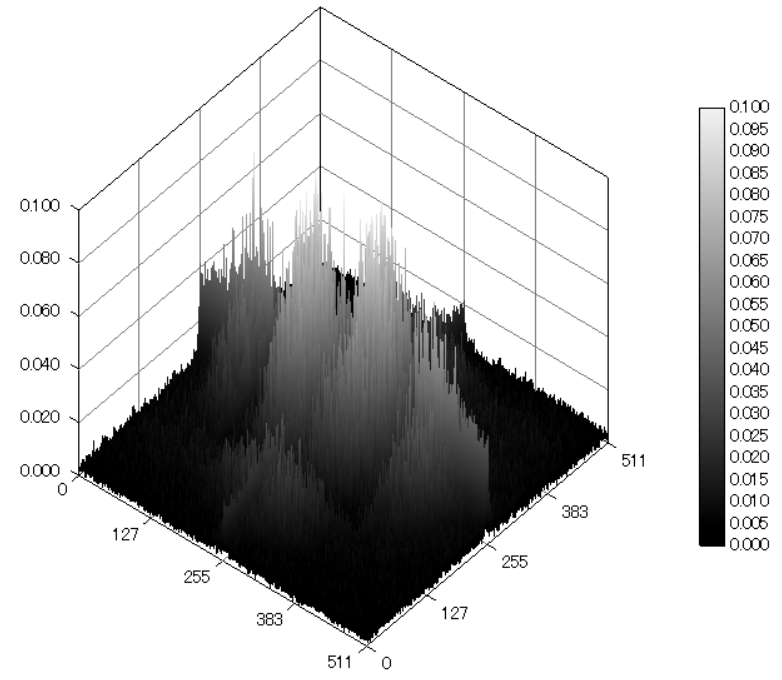

Fig.9. Difference of power spectrum between a cube UM image and the original image.

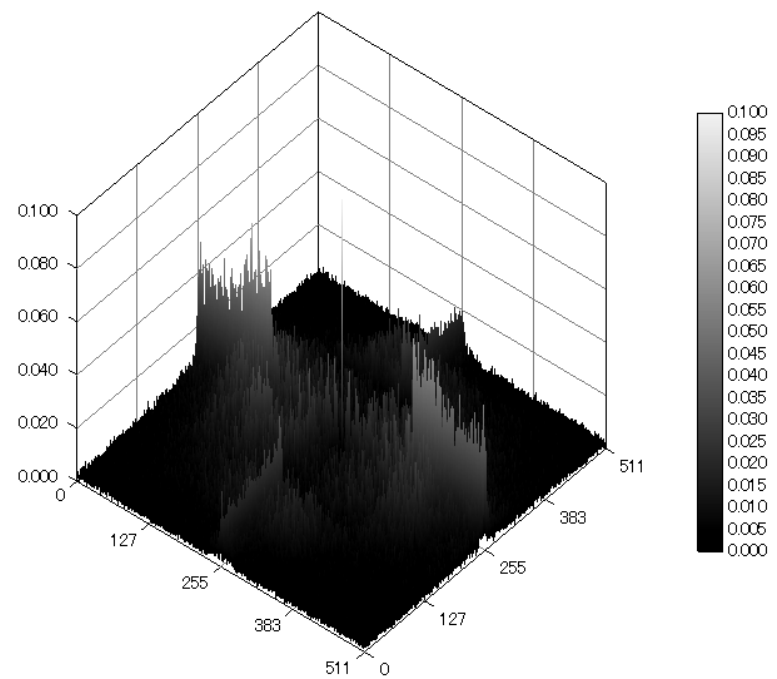

Fig.10. Difference of power spectrum between an image by the proposed method and the original image.

\section{References}

[1] G. Ramponi, "A cubic unsharp masking technique for contrast enhancement", Signal Processing 67, pp. 211 $-222,1998$.

[2] A. Polesel, G. Ramponi, "Image enhancement via adaptive unsharp masking", IEEE Trans. on Processing, vol.9, no.3, 2000.

[3] Ghoshi, "A new signal processing method for video", MMSys '12 Proceedings of the 3rd Multimedia systems Conference, pp. 47-52, 2012. 